\title{
A possible dependence of DF Tauri's photometric activity on the relative orbital positions of the binary components
}

\author{
S. A. Lamzin ${ }^{1}$, S. Yu. Melnikov ${ }^{2}$, K. N. Grankin ${ }^{2}$, and O.V. Ezhkova ${ }^{2}$ \\ 1 Sternberg Astronomical Institute, Moscow V-234, 119899 Russia \\ 2 Ulugh Beg Astronomical Institute and Isaac Newton Institute Uzbekistan Branch, Astronomicheskaya 33, \\ Tashkent 700052, Uzbekistan \\ e-mail: smeln@sai.msu.ru; kn@astrin.uzsci.net; oezh@astrin.uzsci.net
}

Received 20 February 2001 / Accepted 20 March 2001

\begin{abstract}
We constructed a DF Tau historical lightcurve to analyze the character of stellar variability from 1900 to 2000. This time interval exceeds the orbital period $P \simeq 80 \mathrm{yrs}$ of the double system. At least two sections of enhanced photometric activity are present in the lightcurve, with the time interval between them being close to $P / 2$. They correspond to the sections of the DF Tau companion orbit intermediate between apo- and periastron. A decrease in stellar activity occured near the last periastron epoch. We conclude that long-term variations of DF Tau activity are the result of modulation of primary circumstellar disc accretion rate by orbital motion of the companion. We predict that DF Tau activity will increase significantly in the near future.
\end{abstract}

Key words. stars: individual: DF Tau - stars: pre-main sequence - stars: variables - stars: binary stars: circumstellar matter - accretion

\section{Introduction}

DF Tau is a clasical T Tauri star (CTTS): the equivalent width of the $\mathrm{H}_{\alpha}$ line in its spectrum $\sim 50 \AA$ (Herbig \& Bell 1988). DF Tau is a binary (Chen et al. 1990), that consists of two stars of M V spectral type (Ghez et al. 1997). Thiébaut et al. (1995) derived the following set of companion orbital parameters: semi-major axis $a=0.19 \pm 0.03^{\prime \prime}$, orbital period $P=82 \pm 12$ years, excentricity $e=0.8 \pm 0.3$ and inclination angle $i=63 \pm 5^{\circ}$. Ghez et al. (1997) found from Hubble Space Telescope observations that the relative contribution of the companion radiation to the observed spectrum strongly decreases at $\lambda<6000 \AA$ and is less than $10 \%$ at $\lambda \simeq 3000 \AA$. On the other hand DF Tau's optical variability amplitude increases monotonically from $R$ to $U$ band, so one can conclude that the primary is responsible for the variability as well as for the continuum and line emission observed in DF Tau's spectrum (Lamzin et al. 2001).

The inclination angle of the system is relatively small, so it seems rather unlikely that variability occurs due to variations of circumstellar extinction. According to the current paradigm, CTTS activity is the result of disc accretion onto magnetized low mass young star - see e.g. Herbst et al. (1994), Mathieu et al. (2001) and references

Send offprint requests to: S. Lamzin,

e-mail: lamzin@sai.msu.ru therein, - which means that DF Tau's primary has a much more active accretion disc than the companion. It is natural to expect that the primary accretion disc structure will vary as the companion moves along a highly excentric orbit, resulting in a variation of the accretion rate and character of DF Tau's optical variability. The aim of this Letter is to compare DF Tau's optical lightcurve with variations of the distance between the binary system components.

\section{Results}

DF Tau's lightcurve for 1900-2000 is shown in the upper panel of Fig. 1. Photographic magnitudes were used to construct the lightcurve up to the beginning of the 70th year - see Kholopov (1951), Badalyan (1962), Tsesevich \& Dragomirezkaya $(1967,1973)$. Photoelectric $B$-magnitudes observed from the end of the 50th and up to the beginning of the 80th year were adopted from Mendoza (1968), Zajtseva (1970), Zajtseva \& Lyutij (1976a,b), Kuan (1976), Nurmanova (1983), Shaimieva \& Shutemova (1985). These photoelectric and photographic data overlapped in part and are in good agreement. $B$-magnitudes which correspond to the period from the beginning of the 80th year and up to 1997 were adopted from the Herbst database 


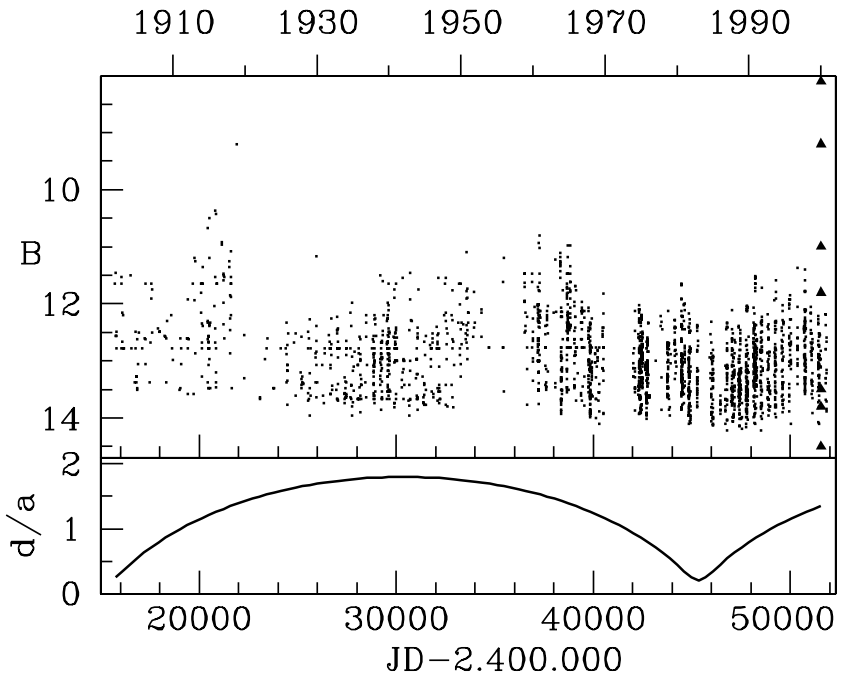

Fig. 1. DF Tau's historical lightcurve based on photographic and photoelectric ( $B$ band) observations (upper panel) and variation of the distance between components of DF Tau with time normalized to the semi-major axis of the companion orbit (low panel). See text for details.

http://www. astro.wesleyan.edu/pub/ttauri (Herbst et al. 1994) and subsequent data are unpublished observations of the authors (K.G., O.E. and S.M.) in the framework of the ROTOR programm - see Shevchenko (1989).

One can see strong light variations within each observational season: flare-like events and quasicyclic variations with a timescale $\sim 7$ days were observed - see Chelli et al. (1999) and references therein. It also follows from Fig. 1 that DF Tau's average brightness and variability amplitude were especially large during the 3-5 year intervals around 1917 and 1963. This feature was discovered by Tsesevich \& Dragomirezkaya (1967), who proposed the hypothesis of the periodic character of DF Tau's activity with a timescale of 44 years - see also Strelkova (1973). Note that this value is close to half of the orbital period found by Thiébaut et al. (1995).

We calculated the variation of the distance between DF Tau's components as a function of time, adopting Thiébaut et al.'s (1995) orbital parameters - the curve normalised to the semi-major axis of the secondary orbit $a$ is shown in the bottom panel of Fig. 1. One can see that stellar activity enhancement occured before and after the epoch of periastron - according to Thiébaut et al. (1995) the latest epoch was $1983 \pm 11$ yr. The second nontrivial feature of the lightcurve is the decrease in stellar activity near the periastron epoch. To demonstrate this fact more clearly we plot in Fig. 2 the $B$ and $V$ lightcurves of the star averaged over observational seasons. In both cases, points can be well approximated with a second order polynomial fit (dashed lines), the minima of which correspond to the end of July, 1987.

It follows from Fig. 1 that decrease in stellar activity during the previous periastron epoch i.e. in the beginning of the 20th century, was less noticeable, but may be the result of low accuracy of the photographic data. Note that

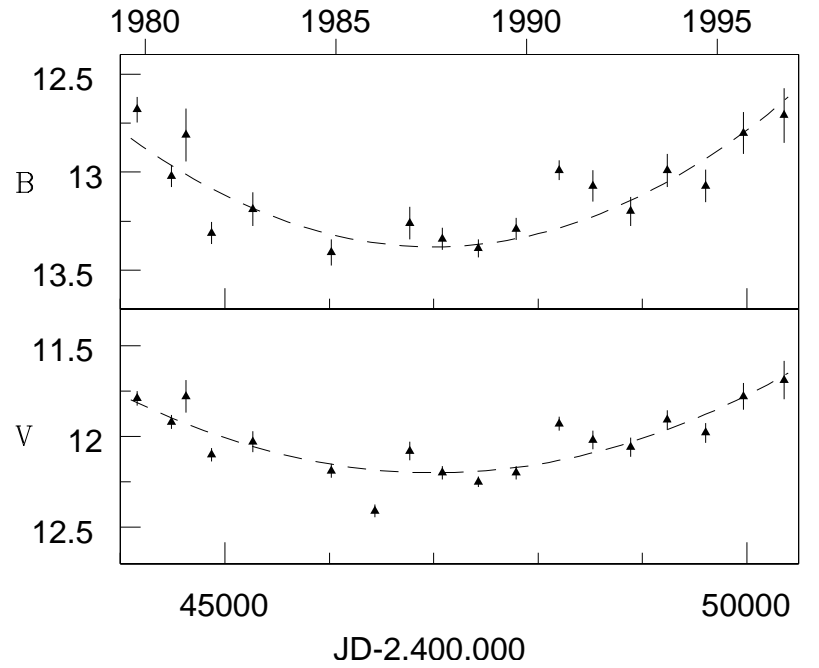

Fig. 2. DF Tau $B$ (upper panel) and $V$ (low panel) lightcurves averaged over observational seasons. The second order polynomial fit is shown with a dashed line. See text for details.

during the subsequent epoch of enchanced activity, stellar brightness reached $m_{\mathrm{pg}}=9.2^{m}$ at JD 2421890.74, i.e. exceeded minimal brightness of this epoch at $4.5^{\mathrm{m}}$. Tsesevich \& Dragomirezkaya (1967) noted that this value is not erroneous. If long term variability of DF Tau indeed has the period equal to the orbital period of the system, one can expect that the new epoch of enchanced activity should occur at the beginning of 21st century. We suggest that a "super flare" $\left(\Delta B \simeq 6.5^{m}\right)$ occured on January 82000 (Li et al. 2001) - see solid triangles in the upper panel of Fig. 1 - and is the first manifestation of the new epoch of DF Tau's activity.

We believe therefore that DF Tau's photometric activity level indeed depends on the distance between the components of the binary system, which means that the accretion rate onto the primary is modulated by the orbital motion of the companion.

\section{Interpretation}

Theory and observations indicate that two kinds of discs can be present in young binary systems: circumstellar (CS) and circumbinary (CB) - see Mathieu et al. (2001) and references therein. Tidal interaction restricts the outer radii $R_{\mathrm{d}}$ of CS discs to $R_{\mathrm{d}} \sim R / 2$ in the case of circular orbit and the $\mathrm{CB}$ disc's inner radius to $2 R$, where $R$ is the distance between binary components (Lubow \& Artimowicz 2001). For a minimum distance between DF Tau's components of $\sim 0.2 a-$ see lower panel of Fig. 1 - the primary CS disc radius cannot exceed this value. If the distance to $\mathrm{DF}$ Tau $\simeq 100 \mathrm{pc}$, then $R_{\mathrm{d}} \lesssim 5$ a.u. It is not known if DF Tau has a CB disc: an upper limit of $M_{\mathrm{d}}<10^{-3} M_{\odot}$ for the mass of DF Tau cold matter was found by Jensen et al. (1994) from submillimiter observations. If the average accretion rate onto the primary is $\dot{M}_{\mathrm{ac}} \sim 3 \times 10^{-9} M_{\odot} /$ yr (Lamzin et al. 2001), then 
this cold matter will be depleted after $t_{\mathrm{ac}}=M_{\mathrm{d}} / \dot{M}_{\mathrm{ac}} \sim$ $3 \times 10^{5}$ years - this value is of the same order as DF Tau's age found by Ghez et al. (1997).

Let us estimate the lifetime $t_{\mathrm{d}}$ of the primary CS disc of radius $R_{\mathrm{d}}$ assuming that it is thin $(H / R \ll 1)$ and has an isothermal atmosphere. If $V_{\mathrm{r}}$ and $T$ are the radial velocity and temperature of the gas near the disc edge, respectively, then it can be derived from the equations of disc accretion theory - see e.g. Shakura \& Sunyaev (1973) and Lipunov (1989) - that

$t_{\mathrm{d}} \sim \frac{R_{\mathrm{d}}}{V_{\mathrm{r}}} \simeq \frac{\sqrt{G M_{*} R_{\mathrm{d}}}}{\alpha \Re T} \simeq \frac{450, \mathrm{yrs}}{\alpha} M_{*}^{1 / 2} R_{\mathrm{d}}^{1 / 2}\left(\frac{30}{T}\right)$,

where $M_{*}$ is stellar mass, $\Re=8.31 \times 10^{7} \mathrm{erg} \mathrm{g}^{-1} \mathrm{~K}^{-1}$ is the universal gas constant, and $\alpha$-Shakura-Sunyaev parameter. $M_{*}, R_{\mathrm{d}}$ and $T$ are expressed in $M_{\odot}$, a.u. and $K$ respectively.

It is usually assumed that $\alpha \sim 0.1-0.001$ in the case of CTTSs - see e.g. Lynden-Bell \& Pringle (1974), Lubow \& Artimowicz (2001). If so, then the circumstellar disc lifetime $t_{\mathrm{d}}$ is in agreement with the $t_{\mathrm{ac}}$ value and it seems resonable to interpret the enchancement of the primary activity as the result of a strong gravitation interaction between the companion and the primary's CS disc during a relatively short period, when the companion passes near the periastron. Apparently, reorganisation of the circumstellar disc structure, which takes some time $\Delta t$, results in the increase of the accretion rate in the innermost disc regions. The $\Delta t$-value should be comparable with the rotation period of the disc external regions (Lipunov 1989) i.e. $\sim 20-30$ years in our case. One can explain in this way the 1917 and the current peak of DF Tau's activity, but the 1960 activity peak is not so clear in the framework of this scenario.

\section{Conclusion}

We analyzed the historical lightcurve of DF Tau which covers a time interval larger than the orbital period $P$ of this binary system. There are two sections of the lightcurve (near 1917 and 1963) separated by a time interval close to $P / 2$ where photometric activity of the primary was especially strong. Apparently, the new cycle of enchanced activity begins now. These activity cycles correspond to the sections of the companion orbit intermediate between apo- and periastron. At the same time DF Tau's activity was minimal near the periastron epoch in the middle of 1980 . We conclude that the CS disc accretion rate onto the primary is modulated in some way by orbital motion of the companion. Further progress will require more accurate information on DF Tau's orbital parameters.

Acknowledgements. We dedicate this article to the memory of V. S. Shevchenko, who was the initiator, the organizer and the chief of the young star monitoring program ROTOR, on the results of which this paper is significantly based. We also would like to thank V. M. Lipunov and V. G. Surdin for helpful discussion, the referee W.Herbst for helpful comments as well as our colleagues from Variable Stars subdivision of Sternberg Astronomical Institute who helped us to search for archival photometrical data.

\section{References}

Badalyan, N. S. 1962, Soobscheniya Byurokanskoi Observatorii, 31, 57

Chelli, A., Carrasco, L., Mujica, R., Recillas, E., \& Bouvier, J. 1999, A\&A, 345, L9

Chen, W. P., Simon, M., Longmore, A. J., Howell, R. R., \& Benson, J. A. 1990, ApJ, 357, 224

Ghez, A. M., White, R. J., \& Simon, M. 1997, ApJ, 490, 353

Herbig, G. H., \& Bell, K. R. 1988, Lick Observatory Bull., 1111

Herbst, W., Herbst, D. K., Grossman, E. J., \& Weinstein, D. 1994, AJ, 108, 1906

Jensen, E. L. N., Mathieu, R. D., \& Fuller, G. A. 1994, ApJ, 429, L29

Kholopov, P. N. 1951, Peremennie zvezdi, 8, 83

Kuan, P. 1976, ApJ, 210, 129

Lamzin, S. A., Vittone, A. A., \& Errico, L. 2001, Astron. Lett., in press

Lipunov, V. M. 1989, Astrophysics of Neutron Stars (Moscow, Nauka)

Li, J. Z., Ip, W. H., Chen, W. P., Hu, J. Y., \& Wei, J. Y. 2001, ApJ, 549, L89

Lubow, S., \& Artimowicz, P. 2001, in Protostars and Planets IV, ed. A. Mannings, A. P. Boss, \& S. S. Russel (Tuscon: University of Arizona Press), 731

Lynden-Bell, D., \& Pringle, J. E. 1974, MNRAS, 168, 603

Mathieu, R. D., Ghez, A. M., Jensen, E. L. N., \& Simon, M. 2001, in Protostars and Planets IV, ed. A. Mannings, A. P. Boss, \& S. S. Russel (Tuscon: University of Arizona Press), 703

Mendoza, E. E. 1968, ApJ, 151, 977

Nurmanova, U. A. 1983, Peremennie Zvezdi, 21, 777

Shaimieva, A. F., \& Shutemova, A. F. 1985, Peremennie Zvezdi, 22, 176

Shakura, N. I., \& Sunyaev, R. A. 1973, A\&A, 24, 337

Shevchenko, V. S. 1989, Herbig Ae/Be stars (in Russian) Tashkent, FAN

Strelkova, A. P. 1973, Astron Tsirc., N. 755

Thiébaut, E., Balega, Y., Balega, I., et al. 1995, A\&A, 304, L17

Tsesevich, V. P., \& Dragomirezkaya, V. A. 1967, Problemi kosmicheskoi fiziki v.2 (Kiev University, Kiev), 110.

Tsesevich, V. P., \& Dragomirezkaya, V. A. 1973, RW Aurigae stars (Naukova Dumka, Kiev), in Russian

Zajtseva, G. V. 1970, Peremennie Zvezdi, 17, 294

Zajtseva, G. V., \& Lyutij, V. M. 1976a, Peremennie Zvezdi, 20,255

Zajtseva, G. V., \& Lyutij, V. M. 1976b, Peremennie Zvezdi, 20,266 